\title{
Self Assertion Modeled as a Network Repertoire of Multi-Determinant Antibodies
}

\author{
Katsuhisa TAKumi $\dagger$ AND Rob J. De Boer \\ Department of Theoretical Biology, Utrecht University, Padualaan 8, $3584 \mathrm{CH}$ Utrecht, \\ The Netherlands
}

(Received on 16 October 1995. Accepted in revised form on 27 March 1996)

\begin{abstract}
We study repertoire selection in a network of natural antibodies that is maintained by stimulatory idiotypic interactions. The natural antibody repertoire develops in an environment of self epitopes to which the self-reactive B cell clones are completely tolerant.

For the modeling formalism, we extend the shape space framework so that each antibody is represented by several randomly chosen shapes. B cell clones are generated stochastically and are removed whenever their density falls below an extinction threshold. The idiotypic interactions are governed by a $\log$ bell-shaped interaction function.

The natural antibody repertoire in the model is formed by percolation: the network is autonomously activated following a point stimulation.

Our main conclusion is that the natural antibody repertoire organizes itself in such a way that most self epitopes are included in the repertoire. We find an over-representation of antibody determinants that are similar to self epitopes. We speculate that the network forms a "smoke screen" covering the somatic self.
\end{abstract}

(C) 1996 Academic Press Limited

\section{Introduction}

The immune system of vertebrates is an ensemble of a very large number of lymphocyte clones. Each clone is characterized by a unique antigen receptor. On a B cell the receptor is the antibody molecule. Because the repertoire of antibody molecules is enormously large, i.e. probably more than $10^{10}$ specificities in the human species (Janeway \& Travers, 1994), any pathogen invading a vertebrate organism will be bound by some of the antibody specificities, and thus evoke an immune reaction. We here consider the repertoire of natural IgM antibodies, i.e. the antibodies that are present before any pathogen has stimulated the immune system (Hooijkaas et al., 1984). These natural antibodies could serve as a primary defense mechanism (Avrameas, 1991) and may play a role in triggering the full-blown immune reaction involving $\mathrm{T}$

$\dagger$ Author to whom correspondence should be addressed. E-mail: takumi@binf.biol.ruu.nl,rdb@alive.biol.ruu.nl. cells and IgG antibodies (Malanchere et al., 1995). We develop a model in which natural antibody repertoire is maintained by stimulatory idiotypic network interactions. We assume that natural antibody repertoire is tolerant to the self antigens, i.e. we assume that $\mathrm{B}$ cell clones with a specificity for any of the organism's own molecules are eliminated by some self tolerance process (Goodnow et al., 1988; Nemazee \& Burki, 1989). We do not consider T cells in our model because the development of natural IgM antibody repertoire seems to be $\mathrm{T}$ cell independent (Malanchere et al., 1995).

Antonio Coutinho and his colleagues also considered an IgM antibody repertoire that is maintained by stimulatory idiotypic network interactions. They coined the term "self assertion" (Coutinho, 1989) for their idea that the idiotypic network is organized around self-reactive B cell clones. Self-reactive IgM antibodies do indeed seem to be a normal component of the natural antibody repertoire (Avrameas, 1991). This view contradicts with existing data that $\mathrm{B}$ cells 
are tolerant to self antigens (Goodnow et al., 1988; Nemazee \& Burki, 1989). Coutinho and his colleagues argue that the self-reactive $\mathrm{B}$ cell clones, by participating in a densely connected idiotypic network, fail to become aggressive towards the self environment. Thus, the network learns what is "self", and self-reactive $\mathrm{B}$ cell clones are no longer dangerous and may even play a role in normal physiology (Coutinho, 1989).

The experimental evidence for immune networks is controversial. Autonomous B cell activity has been studied in mice raised under germ-free conditions (Hooijkaas et al., 1984). IgM antibodies appearing during the early ontogeny of the immune system can bind to each other (Holmberg et al., 1984; Kearney et al., 1987). Experiments suggest that these early antibodies regulate one another (Lundkvist et al., 1989) and influence adult immune responses (Kearney $\&$ Vakil, 1986). Alternatively, the early B cell activity may be due entirely to a special class of $\mathrm{B}$ cells, the $\mathrm{CD} 5^{+}$or Ly1 B cells, which appear early in life before $\mathrm{T}$ cells appear, express IgM, and are multi-reactive (Herzenberg et al., 1986).

Special examples of idiotypic control of self-reactive B cell clones have been reported previously in other models (Neumann \& Weisbuch, 1992a; Stewart \& Varela, 1991; Sulzer et al., 1994; Weisbuch et al., 1993). However, the idiotypic control in these models is not generic because the proposed mechanisms require that all affinities are the same and/or that self antigens are always present in high concentration. In our models which allow to have random affinities, self-reactive clones tend to explode, and thus suppress precisely those specificities that Coutinho supposed would control them (see also De Boer \& Perelson, 1991).

In our model we also find that the idiotypic network organizes itself around the somatic self as argued by Coutinho. For this reason we also use the term self assertion. Our results are, however, almost the reverse of those found by Coutinho and his colleagues since we assume that the B cell clones in our repertoire are completely tolerant to the self antigens. Thus, we cannot explain the empirical reports of the self-reactive $B$ cell clones, and, more importantly, our network cannot be organized around the self-reactive clones. In our model the network is organized around the self antigens, i.e. we find an over-representation of antibody specificities that are self-like (instead of anti-self). However, because our natural antibody repertoire does organize itself around the somatic self, our results are to some extent in agreement with those of Coutinho and his colleagues. Thus, we think that the term self assertion is well chosen and appropriate.

The immune network theory has been studied extensively by means of mathematical models. The model developed in this paper employs the most recent bell-shaped function for B cell proliferation (Sulzer et al., 1996; De Boer et al., 1996), and employs the shape space concept (Segel \& Perelson, 1988) for defining interactions among clones. The word "shape" refers to an abstraction that captures the physical properties of proteins, for example, the geometry of the molecule. Each shape is represented in a finite dimensional space. We consider two complementary spaces between which shapes interact when their coordinates in the spaces are approximately equal. The strength of the interaction diminishes with distance in the shape space.

Our contribution to the shape space formalism is that in our model each antigen receptor is defined as a collection of various shapes in two-dimensional space. In previous shape space models each clone was represented as a single shape only. By lifting this restriction we aim to bring some non-locality into the topology. In our model each clone is able to interact with a set of unrelated shapes. The concept of multiple unrelated shapes is in line with experimental evidence that multireactine $\operatorname{IgM}$ are discriminatory in their binding to antigens (Grandien et al., 1995). Our aim is that as a result of this more global topology of interactions our immune network will inherit some of the pattern recognition properties that normally characterize neural networks (which usually have a very global connection topology).

Our model is based on three assumptions. First, idiotypic interactions stimulate B cell proliferation according to a $\log$ bell-shaped interaction function (De Boer, 1988; De Boer et al., 1996). Second, each antibody is represented by many determinants (shapes). Third, a self tolerance process deletes or anergizes self-reactive B cells (Goodnow et al., 1988; Nemazee \& Burki, 1989). We will show that the three assumptions lead to a natural antibody repertoire that is organized around the self epitopes. We find that natural antibodies that are "suppressed" by strong idiotypic interactions have determinants that are similar to self epitopes. This is why we maintain that the network "asserts the somatic self". We argue that foreign antigens with an epitope that binds to one of the self epitopes will also bind the natural antibodies that mimic the self epitope. Hence the foreign antigens evoke an immune reaction and will be prevented from binding to the self epitopes. 


\section{Model}

In our idiotypic network we consider clones of B cells which can activate each other by idiotypic interactions (Jerne, 1974). Upon activation the clones expand by proliferation. Due to the expansion, the clones may activate other clones, and thus sustain network activity. In our model, B cell clones are produced randomly in bone marrow. We remove clones that are too small (i.e. smaller than a single cell). The only behavior that we allow for the cells of a clone is death (by self-tolerance or normal turnover) or proliferation (by idiotypic activation).

B cells are activated by the cross-linking of their immunologlobulin receptors. We recently derived a $\log$ bell-shaped interaction function by simplifying a model depicting activation by cross-linking with bivalent ligands (Sulzer et al., 1996; De Boer et al., 1996). This function is based on two non-dimensional "fields". The first, i.e. the binding field $h$, is a linear combination of all ligand concentrations weighted by their binding affinity. The second, i.e. the cross-linking field $h_{x}$, weights all ligand concentrations by the product of their binding and cross-linking affinities. Because binding and cross-linking both involve receptor-ligand interactions, we assume that the binding affinity and the cross-linking affinity are proportionally related. The interaction function that we proposed is

$$
f\left(h, h_{x}\right)=\frac{(1+4 \omega) h_{x}}{\omega(1+h)^{2}+h_{x}},
$$

which is bell-shaped on a log scale, $0 \leqslant f\left(h, h_{x}\right)<1$, and where $\omega$ scales the width of the function (De Boer et al., 1996).

The essence of the model presented here is that each clone is represented by several determinants (i.e. idiotopes) in the variable region of the immunoglobulin receptor. We employ the concept of a shape space (Perelson \& Oster, 1979; Segel \& Perelson, 1988) in order to represent each determinant of the variable region by a point in some low-dimensional space. Each idiotype is thus defined as a set of points in shape space. The fields of a determinant are conventionally defined on the local neighborhood of the point corresponding to the exact complementary shape of that determinant. The fields of an idiotype are the sums of the fields of all of its determinants.

We consider two two-dimensional shape spaces of size $L \times L$. The shape of each determinant $\mathbf{s}$ is identified by a vector

where

$$
\mathbf{s} \equiv(c, x, y),
$$

$$
c \in\{0,1\} \text {, and } x, y \in\{1,2,3, \ldots, L\} .
$$

The determinant $\mathbf{s}$ can be interpreted as having a cylindrical shape. The variable $x$ is the radius of the circular base, $y$ is the height, and $c$ designates whether the cylindrical shape is above or below the surrounding molecular surface. The total shape space allows for $2 L^{2}$ different determinants. Let $\mathbf{D}$ denote this set of all possible determinants. We consider any two determinants $\mathbf{s}_{i}$ and $\mathbf{s}_{j}$ in $\mathbf{D}$ to be complementary when their circular-base radius and height are approximately equal, and one protrudes from the surrounding molecular surface and the other is below the surface, i.e. when $\left(x_{i}, y_{i}\right) \simeq\left(x_{j}, y_{j}\right)$ and $c_{i} \neq c_{j}$.

The idiotype of a $\mathrm{B}$ cell clone $b_{\mathbf{I}}$ is defined as a vector of $n$ determinant shapes, i.e. by a $3 \times n$ matrix

$$
\mathbf{I} \equiv\left(\begin{array}{ccc}
c_{1} & x_{1} & y_{1} \\
& \ldots & \\
c_{i} & x_{i} & y_{i} \\
& \ldots & \\
c_{n} & x_{n} & y_{n}
\end{array}\right] .
$$

Setting $L=100$ and describing each clone with $n=4$ determinants we can thus allow for $\left[\left(2 \times 10^{4}\right)^{4} /\right.$ $4 !] \approx 10^{15}$ different clones.

To calculate the fields of each clone we first calculate the fields of each determinant. The binding affinity between two determinants $\mathbf{s}_{i}$ and $\mathbf{s}_{j}$ in $\mathbf{D}$ diminishes as a Gaussian function of the distance from the exact component, i.e.

$g\left(\mathbf{s}_{i}, \mathbf{s}_{j}\right)=\left|c_{i}-c_{j}\right| \exp \left[-\frac{\left(x_{i}-x_{j}\right)^{2}+\left(y_{i}-y_{j}\right)^{2}}{2 \sigma^{2}}\right]$,

where $\sigma$ is the standard deviation of the Gaussian. Note that when $c_{i}=c_{j}$ the affinity $g\left(\mathbf{s}_{i}, \mathbf{s}_{j}\right)=0$, and that the perfect match, i.e. $c_{i} \neq c_{j}, x_{i}=x_{j}$, and $y_{i}=y_{j}$, yields a maximum affinity $g\left(\mathbf{s}_{i}, \mathbf{s}_{j}\right)=1$. The binding fields for a determinant $\mathbf{s}_{i}$ are thus defined as

$$
h_{\mathbf{s}_{i}}=\sum_{\mathbf{s}_{j} \in \mathbf{D}} g\left(\mathbf{s}_{i}, \mathbf{s}_{j}\right) d_{\mathbf{s}_{j}}
$$

where $d_{s_{j}}$ represents the concentration of the determinant with shape $\mathbf{s}_{j}$ in the set $\mathbf{D}$ of all possible determinants. Thus any clone having shape $\mathbf{s}_{j}$ contributes additively to $d_{s_{j}}$.

Here we assume that the cross-linking affinities are proportional to the binding affinities (for a discussion see Sulzer et al., 1996; De Boer et al., 1996). This means that the cross-linking field of the determinants can be written as

$$
h_{x, s_{i}}=\sum_{\mathbf{s}_{j} \in \mathbf{D}} g\left(\mathbf{s}_{i}, \mathbf{s}_{j}\right)^{2} d_{\mathbf{s}_{j}} .
$$


In De Boer et al. (1996) we conclude that if two determinants $\mathbf{s}_{i}$ and $\mathbf{s}_{j}$ have binding affinity $K=g\left(\mathbf{s}_{i}, \mathbf{s}_{j}\right)$, then the cross-linking affinity between the two can best be defined as $J=K^{2}=g\left(\mathbf{s}_{i}, \mathbf{s}_{j}\right)^{2}$.

Thus, the binding and cross-linking fields of a $B$ cell clone $b_{\mathbf{I}}$ are

$$
h_{\mathbf{I}}=\sum_{j=1}^{n} h_{\mathbf{s}_{j}}, \text { and } h_{x, \mathbf{I}}=\sum_{j=1}^{n} h_{x, \mathbf{s}_{j}},
$$

where $j$ runs over all determinants of the idiotype (i.e. all rows of the shape matrix I).

The differential equation describing the growth of a single clone of $\mathrm{B}$ cells is

$$
\frac{\mathrm{d} b_{\mathbf{I}}}{\mathrm{d} t}=b_{\mathbf{I}}\left(p f\left(h_{\mathbf{I}}, h_{x, \mathbf{I}}\right) \frac{\theta}{\theta+b_{\mathbf{I}}}-d\right),
$$

where $p$ is the maximum rate of proliferation, and $d$ is the death rate of $\mathrm{B}$ cells. The term with the $\theta$ parameter defines a maximum population size for a $\mathrm{B}$ cell clone at $b_{\mathbf{I}}=\theta(p-d) / d$. We require a term like this in order to stabilize the steady states (see below). Assuming that activated B cells live about 1 day we set $d=1$. Setting $p=2$ we then obtain a proliferation rate of about one doubling per day. For $p=2$ and $d=1$, the B cell "carrying capacity" is $b_{\mathbf{I}}=\theta$. If $\theta$ is chosen to be 100 , the buffering term is significant for large clones only. (Below we will show that the steady states of the model are located around $b_{\mathbf{I}}=1$.)

The model has a variable structure as a system of ordinary differential equations (ODEs): clones (i.e. ODEs) are constantly produced from bone marrow and are removed by clonal death (i.e., when the last cell dies). Every day the artificial bone marrow creates $M$ new clones with randomly chosen determinants. The initial concentration of such a new clone is $b_{\mathbf{I}}=\varepsilon$, which we consider to be small, e.g. a single B cell. (We typically choose $\varepsilon=0.001$; smaller values gave similar results.) Conversely, clones are removed from the network whenever their concentration drops below the critical concentration of a single B cell, i.e. $b_{\mathbf{I}}=\varepsilon$. Note that according to these rules of creating and deleting clones, new clones which fail to expand are immediately removed from the network. Thus, all clones in our B cell repertoire are sustained by network interactions.

The network is initiated with a set of randomly chosen clones. These initial clones have concentration levels between 0.0 and 1.0. To ensure that the network has a smooth initiation, our artificial bone marrow matures, i.e. $M(t)=M t /\left(k_{t}+t\right)$, where $t$ is the time in days, and $k_{t}=10$ is the day at which the bone marrow output is at half maximal.

\section{IMPLEMENTATION}

The differential equations are solved by Euler integration with a time step of $\Delta t=0.1$. Small time steps gave similar results. At each time interval $\Delta t$ we add and remove clones (i.e. ODEs) according to the rules described above (thus we add $\Delta t M$ clones per integration step). The clones $b_{\mathbf{I}}$ are stored in a hash table. This allows us to check rapidly if a new clone already exists in the network. If so, we cancel its introduction. The fields and the concentrations of the determinants are stored in arrays of size $2 \times L \times L$. Thus, the shape $(c, x, y)$ is directly used for indexing the arrays. At each time interval we check all clones in the hash table to find their concentrations and store all the concentrations of their determinants in the "determinant array". Then the Gaussian equation [eq. (4)] is employed to store the fields of all determinants in the "field array". Finally, the fields of all the clones $b_{\mathrm{I}}$ are computed by sampling the field array for each determinant in $b_{\mathbf{I}}$. Having the fields and $b_{\mathbf{I}}$ we solve eqn (8) for a time step $\Delta t$.

For computational purposes we truncate the Gaussian function to

$$
\hat{g}\left(\mathbf{s}_{1}, \mathbf{s}_{2}\right)=\left\{\begin{array}{cl}
g\left(\mathbf{s}_{1}, \mathbf{s}_{2}\right) & \text { if } g\left(\mathbf{s}_{1}, \mathbf{s}_{2}\right)>\kappa \\
0 & \text { otherwise }
\end{array}\right.
$$

where we typically choose $\kappa=0.1$. This does not affect the results since we know that models based on eqn (1) have a "minimum affinity" below which clones cannot respond. Smaller values of $\kappa$ did indeed give similar results. We typically choose $\sigma^{2}=4.5$. Then, by the truncation with $\kappa=0.1$, the radius of the region covered by Gaussian function is $r=6$. The total area covered by the Gaussian neighborhood is 61 elements in the array (which is $0.3 \%$ of the total shape space).

\section{PARAMETER VALUES}

We used the following parameter values to produce all the results reported in this paper; $L=100, p=2.0$, $d=1.0, \quad \omega=0.1, \quad \theta=100, \quad \varepsilon=0.001, \quad k_{t}=10$, $\sigma^{2}=4.5, \quad \kappa=0.1, \quad$ bone marrow recruitment rate $=4 \times 10^{4}$ clones per day. We chose few initial clones, i.e. $N_{0}=10$, for a multi-determinant network to illustrate that such networks fill the shape space by percolation. We chose $N_{0}=10^{4}$ for a single-determinant network to illustrate that such networks do not percolate. 


\section{Results}

\section{TWO INTERACTING CLONES}

The simplest network in our model consists of two clones of B cells, having a binding affinity $K=1$ at one determinant only. This need not be studied in shape space because we can call the first clone $b_{1}$ and the other $b_{2}$ with

$$
h_{1}=K b_{2}, \quad h_{x, 1}=J b_{2}, \quad h_{2}=K b_{1}, \quad h_{x, 2}=J b_{1},
$$

where the interaction strength $J=K^{2}$. These four fields with two ODEs defined by eqn (8) define a simple two-clone network. This network is very similar to the two-clone model analysed by De Boer et al. (1996) except that it includes a buffering term but excludes continuous bone marrow influx. To set the stage for the more complicated shape space
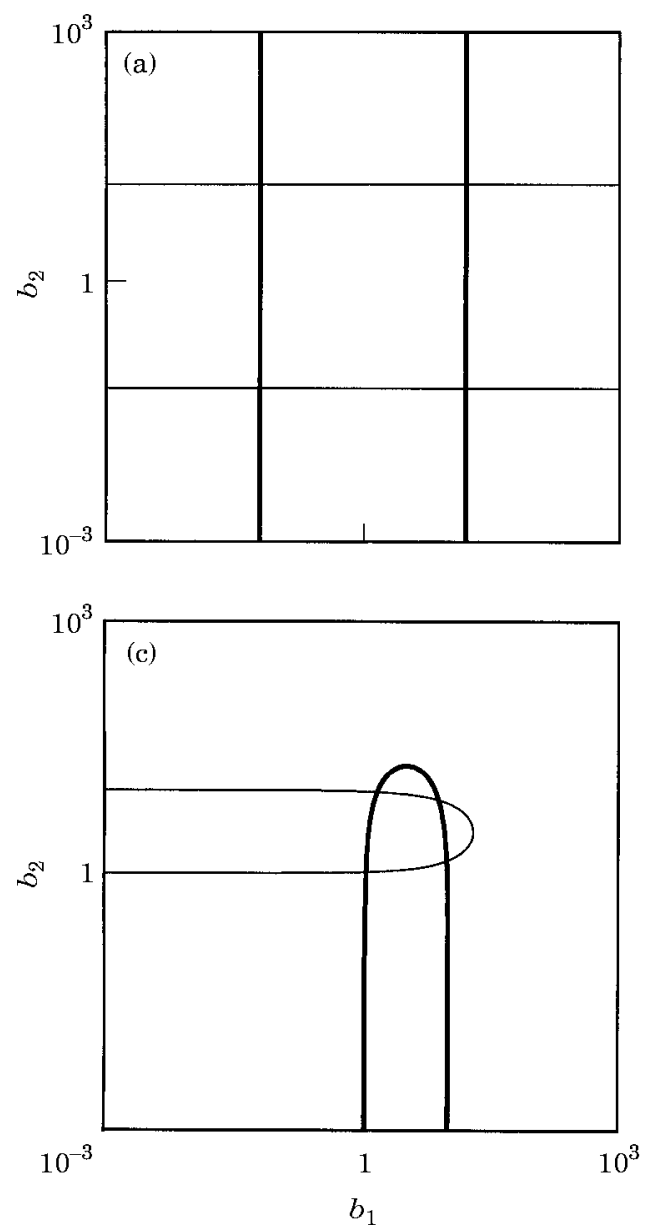

models presented below, we first study this two-clone network by phase plane analysis.

We first explain why the buffering term is required. Letting $\theta \rightarrow \infty$ we show in Fig. 1(a) that the nullclines are straight and intersect perpendicularly in four steady states. An additional steady state is the virgin state $b_{1}=b_{2}=0$. In the non-trivial steady states, a clone with a low equilibrium field can be called "immune" because increasing its field increases its rate of proliferation. A clone with a high equilibrium field also proliferates, but when it is further stimulated it decreases its rate of proliferation. Such a clone is then said to be "suppressed" (De Boer, 1988; Weisbuch et al., 1990; De Boer et al., 1993a, b; see also the Appendix).

The problem with the two asymmetric steady states $\left(b_{1} \neq b_{2}\right)$ in Fig. 1(a) is that they are "neutrally stable", i.e. the eigenvalues of the Jacobian are purely
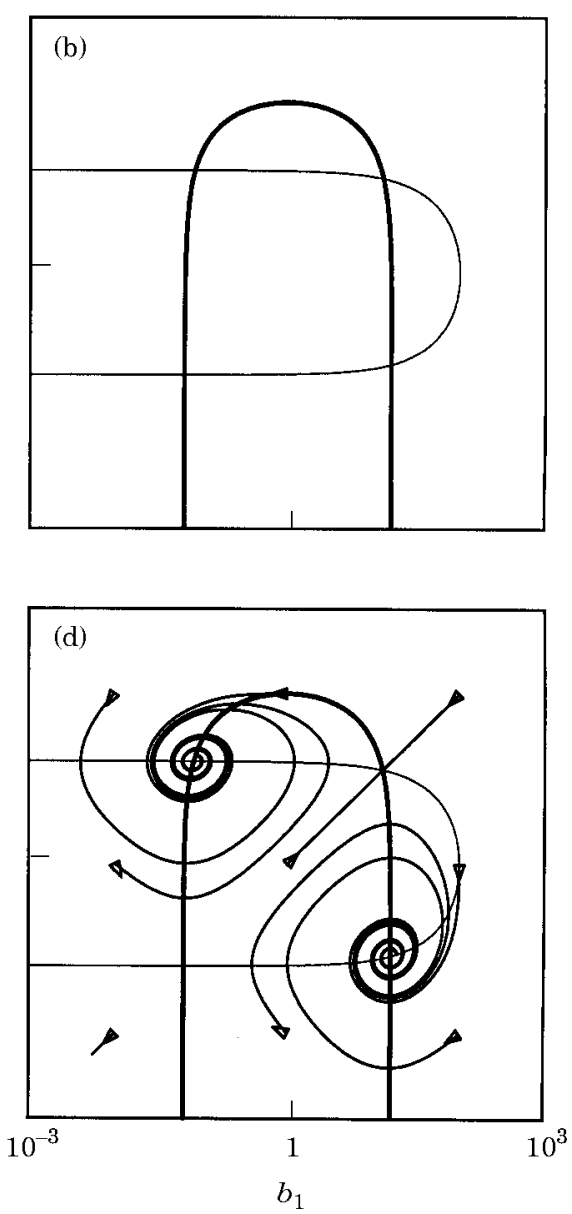

FIG. 1. Phase planes of the two-clones network. The light lines are the $b_{1}$ nullclines. The dark lines are the $b_{2}$ nullclines. The original system is structurally unstable since it has purely imaginary eigenvalues (a). We need the buffering term to make the system stable (b). A property of the proliferation function is that four equilibria come close together as affinity decreases (c) and eventually disappear. Trajectories are computed by Euler integration (d). We used the following parameter values to produce and only to produce Fig. 1(a) through 1 (d). $K=1.0$, and $\theta=\infty$ for (a). $K=1.0$, and $\theta=100$ for (b). $K=0.3$, and $\theta=100$ for (c). $K=1.0$, and $\theta=100$ for (d). The parameters $p, d$, and $\omega$ are listed in the Parameter values section in the text. 
imaginary. Therefore, with $\theta \rightarrow \infty$ the model is not structurally stable. We solve the problem by adding some curvature to the nullclines (in previous models the curvature was due to a non-trivial virgin state). By setting $\theta=100$ in the buffering term, we obtain the curvature of the nullclines for large clone sizes shown in Fig. 1(b). Now the asymmetric steady states become stable spiral points [Fig. 1(d)]. The two symmetric steady states are saddle points. The four steady states remain qualitatively the same as those in Fig. 1(a) but the model is now structurally stable.

For our standard parameters we find the four steady states are centered around $b_{1}=b_{2}=$ $h_{1}=h_{2}=1$. For the binding affinity $K=1$ the steady states are located around $\left(b_{1}, b_{2}\right) \simeq(16,0.1)$ $\left(b_{1}, b_{2}\right) \simeq(0.1,16)$ [see Fig. 1(b)]. The trajectories in Fig. 1(d) are computed by Euler integration with $\Delta t=0.1$. The trajectories are smooth and are similar to those computed with variable time step integration (not shown).

The effect of decreasing the affinity of the idiotypic interaction is studied in Fig. 1(c). For $K=0.3$ the four steady states still exist, but the regions where clones can increase in concentration have become smaller [Fig. 1(c)]. Decreasing the binding affinity still further leads to a saddle-node and a pitchfork bifurcation around $K=0.22$ by which the four states disappear (cf. De Boer et al. 1993a, b). We conclude that $K \simeq 0.2$ defines a minimum binding affinity below which clones cannot interact. This is why truncating our Gaussian equation [eq. (4)] at a binding affinity of $\kappa=0.1$ has no effect on the model behavior.

\section{Network Behavior}

Having set a baseline we now consider networks with many clones. For convenience, we call networks in which each receptor is represented by one determinant only the "single-determinant model". Likewise, we call networks in which each receptor is represented by many determinants the "multi-determinant model". We first consider networks without self antigens.

\section{PERCOLATION}

In immune networks, percolation means the spreading of idiotypic activation from a local interaction (De Boer \& Hogeweg, 1989; Weisbuch et al., 1990; Neumann \& Weisbuch, 1992b). We recently derived the interaction function [eqn (1)] which selects high affinity interactions out of a random set of clonal interactions. By selecting high affinity interactions, the interaction function allows for localized states in shape space models and in random networks (De Boer et al., 1996; see also Fig. 2). The random networks will either percolate or have localized behavior depending on parameter values. For instance, if $\mathrm{B}$ cell clones are produced stochastically, random networks percolate. Localized attractors exist but are typically not attained due to the transients induced by the stochasticity. For the multi-determinant model, we therefore expect percolation to be the dominant behavior because it produces B cell clones stochastically and has several high affinity connections per clone.

We observe percolation in the multi-determinant model but not in the single-determinant model. This is shown in Figs 2 and 3 which depict the network repertoire in shape space by color indexing of the concentration of all determinants $\mathbf{s}=(c, x, y)$. In the single-determinant model, i.e. for $n=1$, we need a large number of initial clones to cover ultimately most of the shape space. Starting with $10^{4}$ initial clones, the repertoire forms many localized clusters (Fig. 2). The time plot [Fig. 2(d)] shows that a large fraction of

FIG. 2. The antibody repertoire of the single-determinant model in shape space. The repertoire is visualized by color indexing of the concentrations of all determinants. The colors are scaled logarithmically. A bright color stands for a high concentration and a dark color for a low concentration. The shape space with $c=0$ is colored in red (a) and the shape space with $c=1$ is colored in green (b). Plate (c) is a superposition of (a) and (b). Plate (d) is a time plot of the middle section in (c) from day 0 to day $10^{3}$. We start the simulation with $10^{4}$ random antibodies. For the other parameter values, see the Parameter values section in the text.

FIG. 3. The antibody repertoire of the multi-determinant model $(n=4)$ in shape space. We start the simulation with ten random antibodies. For a description of the plates and other parameter values, see the legend in Fig. 2.

FIG. 4. The repertoire shown in Fig. 3(a) and (b) is decomposed into functional and dummy determinants. A determinant is called functional if it receives a field of more than 0.005 , otherwise it is called dummy. The functional determinants are shown in panels (a) and (b). The dummmy determinants are shown in (c) and (d). The threshold 0.005 is chosen to be small enough compared with the concentration of suppressed clones, which is approximately equal to 0.1. Setting the threshold lower hardly changes the figure.

FIG. 5. The antibody repertoire develops around the self antigens. Epitopes of self antigens are marked by blue crosses. Antibody determinants identical to self antigens are also present in the panels but are hidden by the blue crosses. There are 40 epitopes of self antigens covering maximally $40 \times 0.3 \%$ of shape space. The repertoire of the $n=1$ network is shown in panels (a) and (b). The repertoire of the $n=4$ network is shown in panels (c) and (d). Snapshots are taken at day $10^{3}$. There are 1044 clones in the $n=1$ network and 3829 clones in the $n=4$ network. 

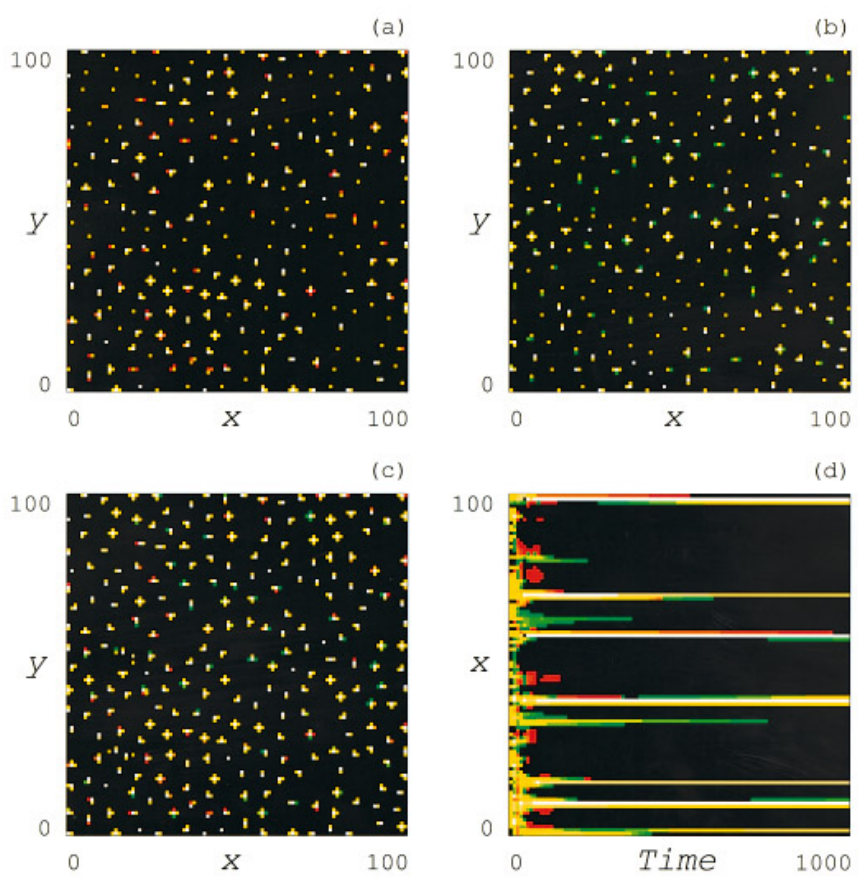

FIG. 2.

(a)
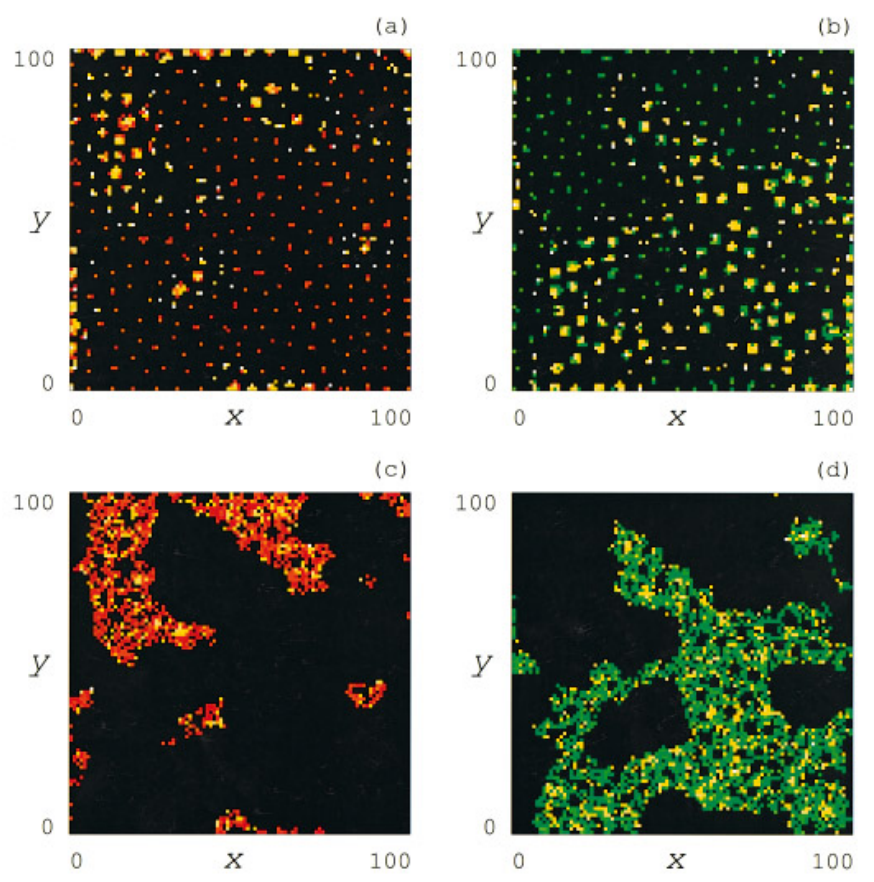

FIG. 4.

Katsuhisa Takumi and Rob J. De Boer
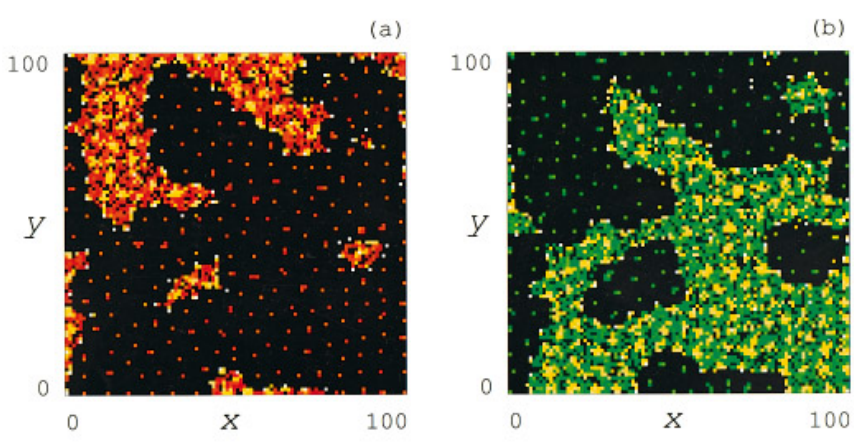

(c)
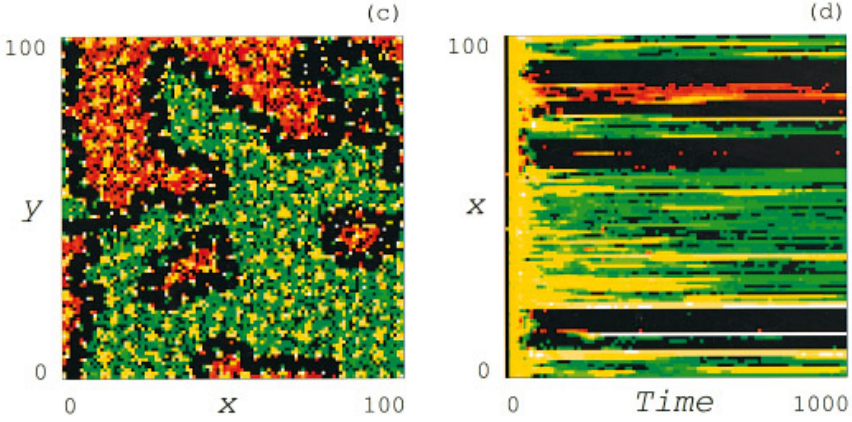

FIG. 3.

(a)
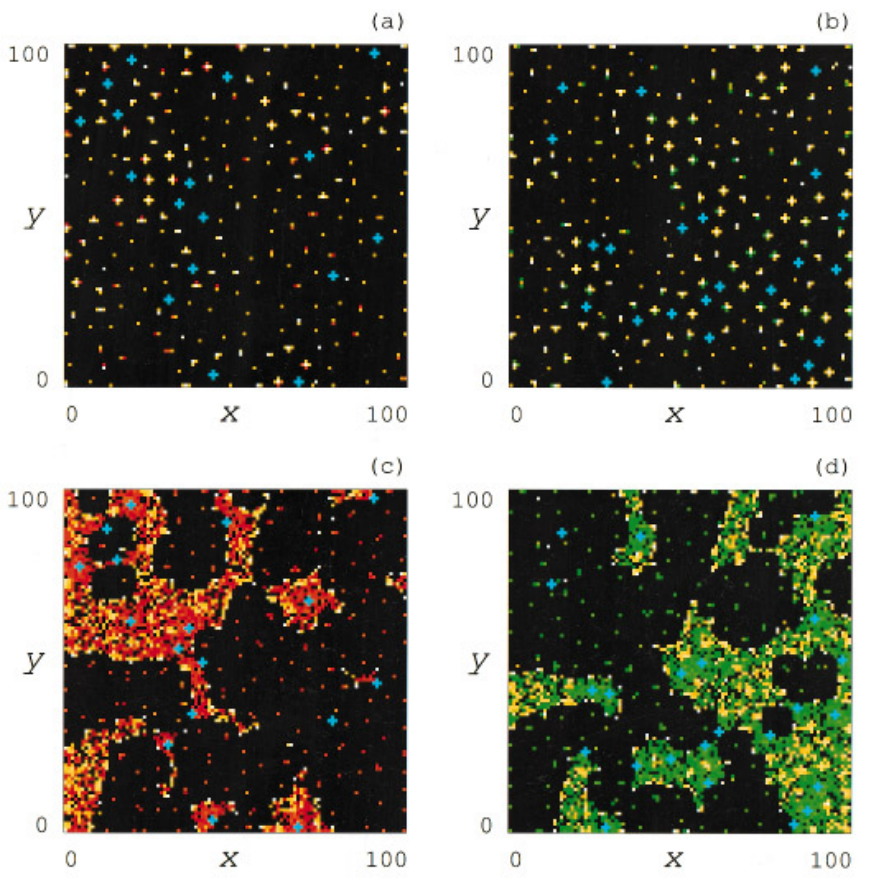

FIG. 5. 
clones present at time zero becomes extinct quickly. In the multi-determinant model, i.e. for $n>1$, the repertoire forms large connected structures starting with only ten initial clones (Fig. 3). The time plot [Fig. 3(d)] reveals that in the middle section of the shape space the idiotypic activation spreads from two initial spots at time zero.

\section{SINGLE-DETERMINANT MODEL}

Because we observe no percolation in the $n=1$ case, we can say that the model behaves similarly to a previous one-dimensional shape space model (De Boer et al. 1996). In the current model, the repertoire typically evolves to "clusters". We observe clusters consisting of five immune clones all supported by one suppressed clone (Fig. 2). The immune clone in the middle is the largest and interacts with the suppressed clone with which it has maximum affinity.

To understand the cluster formation, consider a pair of clones, one immune and the other suppressed, interacting with each other with maximum affinity. Assume that the two clones are in equilibrium. For the large immune clone, the buffering term is significant so that $f\left(h, h_{x}\right)>d / p$. Thus, because clones in the immediate neighborhood of the immune clone have fields similar to those of the immune clone, they can increase in concentration. Conversely, for the small suppressed clone, the buffering term is not significant and $f\left(h, h_{x}\right) \approx d / p$. Hence clones in the immediate neighborhood have $f\left(h, h_{x}\right)<d / p$ and cannot increase.

Although clusters are formed locally, they are not randomly spread over shape space. In Fig. 2, we do observe a large domain in which clusters of the same color group together. Elsewhere we study the domain formation by linear stability analysis (Noest et al., 1996). From our initial condition, the system rapidly grows to a state where all clones are large with small fluctuations in clone's concentrations, i.e. approaches the upper saddle point in Fig. 1(b). Modeling the spatial fluctuations over the shape space as cosine waves of various frequencies and computing the eigenvalues of the system of the B cell clones, we found that the system is unstable under low frequency sinusoidal perturbation. A superposition of low frequency cosine waves produces a large domain in the shape space where fluctuation is positive for one color. Hence clones of the same color become immune in the large domain (Noest et al., 1996).

MULTI-DETERMINANT MODELS: FUNCTIONAL AND DUMMY

An important concept for understanding the behavior of multi-determinant models is that of "functional" and "dummy" determinants. We will show below that the field which a clone in the network receives comes only from a subset of its determinants. These determinants are called the "functional" determinants of the clone. The remainder, i.e. those determinants which hardly contribute to the clone's field, are called the "dummy" determinants of the clone. In other words, dummy determinants are those determinants which are "parked" at places where the field is low.

If we start with a few clones, the percolation causes a rapid expansion of the repertoire [see around time zero in Fig. 3(d)]. In a short time, the shape space is fully occupied by a mixture of random clones. Most of the clones are then eliminated from the network repertoire because they receive too high a field, but a few clones stay in the network. The determinants expressed by the clones in the network form large red and green areas extending over shape space [Fig. 3(a-c)]. In order to understand the pattern formation, we plot the functional and the dummy determinants separately in Fig. 4. On the level of the functional determinants [Fig. 4(a) and (b)], clusters of the same color group together by the same pattern formation mechanism that we explained above for the $n=1$ case (Noest et al., 1996). Thus, the functional determinants are typically complementary to each other. The novel property of multi-determinant networks is that the dummy determinants fill up the space between the functional determinants which results in the large red and green areas [Fig. 4(c) and (d)].

In the multi-determinant model there is an additional positive feedback mechanism reinforcing the domain formation. Consider a pair of functional determinants, one belonging to an immune clone and the other belonging to a suppressed clone. The immune clone's determinant evokes a large high field region around the suppressed clone's determinant. Conversely the suppressed clone's determinant evokes a small low field region around the immune clone's determinant. Thus, a novel clone appearing from the bone marrow with a determinant close to the immune clone's determinant will receive a very low field, i.e. this new determinant will be dummy. The accumulation of such a dummy determinant exerts an even stronger field around the suppressed clone's determinant. This stronger field will ultimately decrease the low field around the immune clones and hence invites more dummy determinants. By means of this positive feedback mechanism, domains of the same color enlarge.

We view the presence of determinants [Fig. 3(a-c)] as a natural antibody repertoire maintained by the network. Once it is formed, the repertoire is stable over time [Fig. 3(d)]. 


\section{DOUBLE TOLERANCE}

We now introduce self antigens into our single-determinant model. We incorporate the third assumption that a self tolerance process deletes self-reactive B cells. Although natural antibodies can be self-reactive (Avrameas, 1991), there is experimental evidence that B cells are tolerant to self antigens (Goodnow et al., 1988; Nemazee \& Burki, 1989). In fact self tolerance is required in our model because without it the self-reactive B cell clones will proliferate strongly in response to those self antigens that are present at stimulatory concentrations.

The assumptions that (i) the natural antibody repertoire is maintained by stimulatory network interaction, and that (ii) self antigens generate self tolerance, imply an unexpected form of "double tolerance". Because self epitopes shoot holes in the repertoire, antibodies with determinants that are similar to self epitope will hardly be stimulated. This is disadvantageous. The natural antibody repertoire should cover most of the shape space in order to initiate immune responses to most foreign antigens.

We implement self antigens by randomly seeding self epitopes in the shape space before any B cell is present. The self epitopes persist at a fixed (arbitrary) concentration. We assume that $\mathrm{B}$ cell clones that have a binding affinity greater than $\kappa$ to any of the self epitopes are tolerized (i.e. deleted) by some self-tolerance process. Self antigens thus shoot holes in the repertoire. We typically choose $\kappa=0.1$. (The same value of $\kappa$ is used for truncating the Gaussian, see the implementation section.)

In Fig. 5(a) and (b), we illustrate the repertoire formation for the $n=1$ network. Self epitopes are marked by blue crosses. In the single-determinant model, all crosses are isolated from the repertoire [Fig. 5(a) and (b)]. Thus, the single-determinant model suffers from double tolerance. In fact, double tolerance is implied in all previous immune network models. This is a natural consequence of network interaction and the self tolerance process.

\section{SELF ASSERTION}

We show that the multi-determinant model does not suffer from double tolerance. When we change the single parameter $n$, the number of determinants per antibody receptor, double tolerance disappears. Instead, the natural antibody repertoire arising in the multi-determinant model "asserts" the self antigens.

In Figs 5(c) and (d), we illustrate the repertoire formation around the self antigens for the $n=4$ network. Self epitopes are also marked by blue crosses. Figure 5(c) and (d) show that most of the crosses are included in the repertoire. Thus, epitopes of self antigens are surrounded by natural antibody determinants of similar shapes. This is what we mean by self assertion.

The determinants surrounding the epitopes have to be dummy determinants. This is because they cannot receive a field from the complementary region where all B cells are tolerized. We conclude that the self antigens are embedded in dummy determinants with shapes similar to its epitopes. In addition, we will show below that the dummy determinants belong primarily to suppressed clones. Thus, self antigens are largely asserted by suppressed clones. This can also be seen in Fig. 5(c) and (d) where epitopes of self antigens are surrounded by determinants of a darker
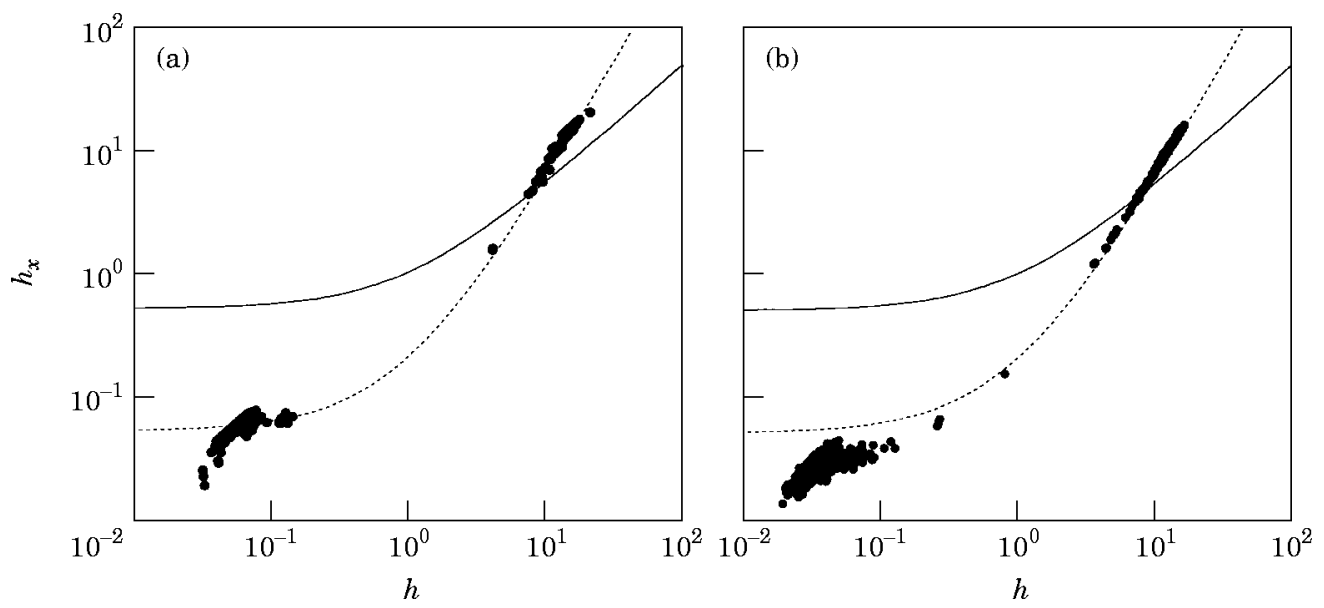

FIG. 6. Plots of the cross-linking field $h_{x}$ and the binding field $h$ for all clones in the $n=2$ network (a) and the $n=4$ network (b). The relation between the two fields is such that they lie on the dotted curve [eqn (11)] when a clone is in equilibrium. The solid curve is the suppressive condition [eqn (A.4)] based upon the two fields. Clones above the solid curve are suppressed and below the curve are immune. According to the condition, approximately $60 \%$ of the total population is suppressed in the multi-determinant model of panels (a) and (b). But only $30 \%$ is suppressed in the single determinant model (not shown). The data refer to day $10^{3}$. 

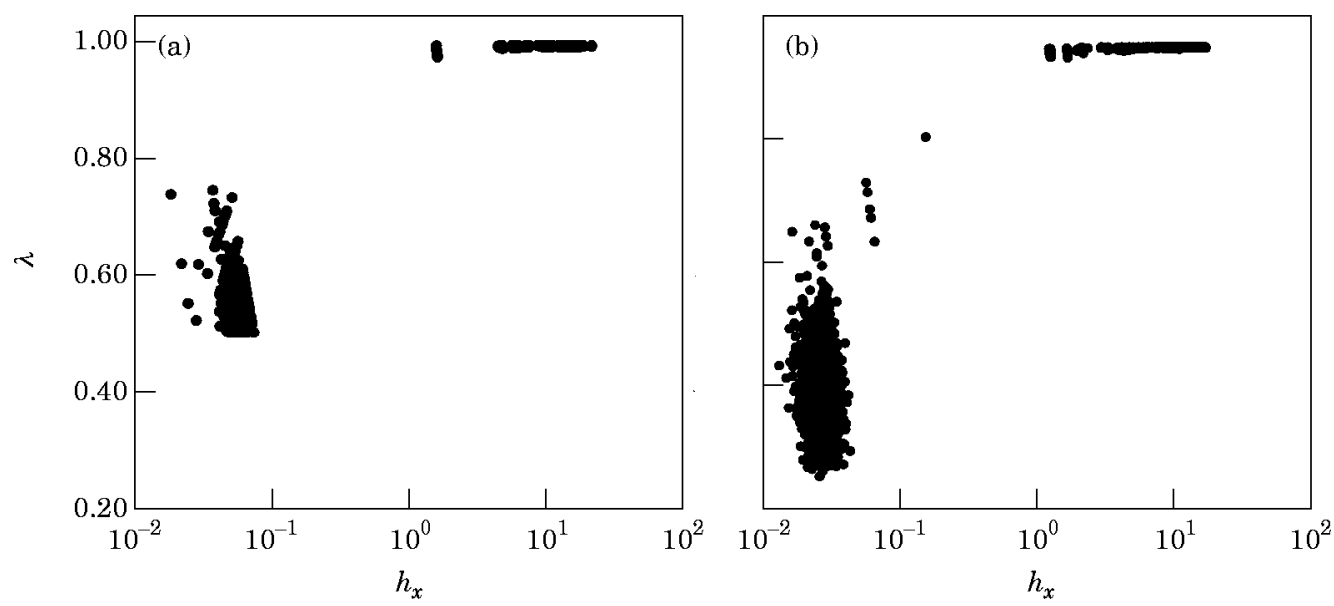

FIG. 7. For each clone, we define $\lambda$ as a measure of the contribution made by the determinant with the largest field (see also the Network statistics section). We plot $\lambda$ values against $h_{x}$ for each clone in the $n=2$ network (a) and the $n=4$ network (b). Suppressed clones (the dots for which $h_{x} \simeq 10$ ) have $\lambda \simeq 1$, indicating that they have only one functional determinant. Immune clones, i.e. $h_{x} \simeq 10^{-1}$, have $\lambda$ around $1 / n$. Hence many of their determinants are functional. The data refer to day $10^{3}$.

color, which indicates that the determinants do indeed belong to suppressed clones.

A self epitope is only asserted by the network if it is not complementary to another self epitope. Two complementary self epitopes typically evoke double tolerance. When the number of self epitopes is sufficiently small with respect to the size of shape space, the repertoire develops in such a way that most self antigens have their epitopes asserted by suppressed clones [Figs 5(c) and (d)].

\section{Network Statistics}

We here provide some evidence for the concepts used in the previous section. First, we show that most clones in the network are in equilibrium. For a clone in equilibrium, eqn (8) is zero. Thus, its binding and cross-linking fields satisfy the condition,

$$
h_{x}=\frac{d \omega(1+h)^{2}}{p(1+4 \omega)-d},
$$

assuming that the buffering term for the clone is approximately one. In Fig. 6, we plot the binding and the cross-linking fields for each clone in the network. We see that the fields lie near the curve defined by eqn (11) (dotted curve). Thus, most clones in the network are in equilibrium. The fact that low-field clones are located below the dotted curve in the $n=4$ case is because (i) low-field clones tend to be large so that the buffering term in eqn (8) is significant, and (ii) part of low-field clones are decreasing due to a lack of stimulatory interaction, i.e. are indeed not in equilibrium. The solid curve in Fig. 6 is the two-field suppression condition that we derive in the Appendix [eqn (A.4)]. Above the solid curve clones are suppressed, below it clones are immune. There are few clones however which lie below the curve but nevertheless have a large binding field, i.e. $h>1.0$. We have checked that they have low affinity interactions and hence do not comply to the simplified suppression condition [eqn (A.4)], i.e. they are suppressed according to eqn (A.3).

\section{FUNCTIONAL AND DUMMY}

The concept of functional and dummy determinants is quantified in Fig. 7. For each clone in the network, we determine the cross-linking fields $h_{x, s_{j}}$ [eqn (6)] received via each determinant $\mathbf{s}_{j}$ of the clone and its total cross-linking field $h_{x}=\sum_{j=1}^{n} h_{x, s_{j}}$ [eqn (7)]. Then we define $\lambda=\max _{j} h_{x, s_{j}} / h_{x}$ as a measure of the contribution made by the determinant with the largest field. Thus if $\lambda \approx 1$ the clone has only one functional determinant. In Fig. 7 we express $\lambda$ as a function of $h_{x}$ for all clones in the network. The dots around $h_{x} \approx 10^{-1}$ are immune clones, whereas those around $h_{x} \approx 10$ are suppressed. This is consistent with the suppressive condition which we derive in the Appendix. Suppressed clones almost always have one functional determinant only, i.e. $\lambda \approx 1$. Immune clones have a considerably lower $\lambda$. Thus immune clones have long range interactions: they obtain their fields from several regions in shape space. (We have checked that the determinants of immune clones are not close together in the shape space. But this is not shown.)

There is a simple reason why immune and suppressed clones have different $\lambda$ values. Since immune clones become larger with increasing fields, immune clones are replaced by clones having more functional determinants. Suppressed clones on the other hand, become smaller with increasing fields, so they are replaced by clones having more dummy determinants. In summary, the network seems to sort itself so that immune clones have only functional 
determinants and suppressed clones have one functional determinant, the rest being dummy.

\section{OTHER NETWORK PROPERTIES}

The results presented in this paper are obtained from simulations run up to day $10^{3}$. We choose a thousand days because the observed patterns (Figs 2 and 3) and the network statistics (Figs 6 and 7) change only slightly over longer time. An exception is the number of clones in the network. Initially the number changes as in the bitstring model (De Boer et al., 1991). A longer simulation $(n=2)$, run up to day $10^{4}$, shows however that the network size keeps on increasing. The increase is caused by clones sharing more and more of their determinants and reducing their concentrations appropriately. We do not perceive a similar increase for the $n=4$ over a period of up to $10^{4}$ days. However, we expect the network size to increase if the simulations are run for an even longer time.

\section{Discussion}

Our multi-determinant idiotypic network of natural antibodies demonstrates self assertion. Incorporating complete self tolerance, we find that the epitopes of self antigens become "embedded" in a repertoire of natural antibodies of intermediate concentration. In our model these natural antibodies are the "dummy" determinants of suppressed clones. Thus, we find an over-representation of self-like antibodies in the natural antibody repertoire.

The IgM natural antibody repertoire possibly plays a role as a primary defense mechanism (Avramees, 1991) and in the initiation of a full-blown immune response involving helper $\mathrm{T}$ cells and $\mathrm{IgG}$ antibodies (Malanchere et al., 1995). If this is the case, then the antibody repertoire of our model network would select some epitopes of a foreign antigen to trigger a full-blown immune response, i.e. $\mathrm{T}$ cell dependent immune response. Due to its over-representation of self-like antibodies, our natural antibody repertoire selects the anti-self epitopes of foreign antigens to trigger a full-blown immune reaction.

We speculate that the natural antibody repertoire is advantageous because the anti-self epitopes of foreign antigens are the most vital ones for the pathogen and the most dangerous ones for the host organism. We expect the pathogen to use the anti-self epitope in order to interact with the host physiology, thereby infecting the host. In the previous immune network models self antigens evoke double tolerance, which implies that the anti-self epitopes of pathogens will not be selected for triggering an immune reaction.
In our multi-determinant model, however, foreign anti-self epitopes are selected for triggering immune reactions. Also in our model, the self-like natural antibody might function as a "smoke screen" by preventing the most vital epitopes of the foreign antigens from interacting with the host physiology.

The weakness of this speculative argument involving the host physiology is that the self antigens should also bind other self antigens to form the physiology of the organism. Therefore, many self epitopes interact with complementary self epitopes on other molecules. Due to the complete self tolerance mechanism, such a complementary pair of self epitopes shoots two complementary holes in the repertoire, i.e. evokes double tolerance (cf. Weisbuch et al., 1993). Those foreign determinants that interact with either of the two self epitopes will therefore escape immune detection. This problem is a natural consequence of the self tolerance process, and allows one to compute the minimum size of the antibody repertoire to ensure that other determinants of the pathogen will evoke an immune reaction (De Boer \& Perelson, 1993c). Because most self molecules are large complex proteins, they will also express many self epitopes that do not interact with the physiology (Clackson \& Wells, 1995). We could again call such epitopes the "dummy self epitopes", as opposed to the "functional self epitopes" that have a function in the physiology. Pathogens that are evolving epitopes to interact with self could select either dummy or functional self epitopes. The former selection would be even more likely if the dummy self epitopes did indeed outnumber the functional ones. In our multi-determinant model, the pathogens interacting with dummy self epitopes will always trigger an immune reaction and/or are fooled by mistaking the natural antibody for the self antigen.

In our current model we had to incorporate an explicit self tolerance mechanism to avoid the explosion of self reactive clones. It would be very interesting if our multi-determinant immune network had been able to account for self tolerance by itself. Thinking that self reactive clones could become suppressed because they receive a field from both the self antigens and from the network, we have searched for parameter regions where an explicit tolerance mechanism would not be required. Thus, we thought that the additional stimulation from self antigens would facilitate the suppression of self reactive clones. Although our hypothesis worked occasionally, we have been unable to obtain a robust self tolerance in which self reactive clones generally become suppressed.

Because we extend the shape space formalism with 
multi determinants per idiotype or per self antigen, our model overcomes the problem of double tolerance that is ubiquitous in the previous network models. The fact that our model does not have double tolerance enlarges the diversity of the natural antibody repertoire and enables the host organism to immunologically defend itself against a larger variety of pathogens. Additionally, if the natural antibody repertoire did indeed initiate a full-blown immune response, the self assertion in our model ensures the triggering of an immune reaction to the most vital epitopes of a foreign intruder.

K. Takumi was supported by the Life Sciences Foundation (SLW) of the Netherlands Organization for Scientific Research (NWO). This work was finished while we visited the Santa Fe Institute. We thank Alan Perelson and the Santa Fe Institute's Theoretical Immunology Program supported by the Joseph P. and Jeanne M. Sullivan Foundation. We thank Bernard Sulzer for improving the general suppression condition of Eq. (A.3). We are grateful to Ms S. McNab for linguistic advice.

\section{REFERENCES}

Avrameas, S. (1991). Natural antibodies: from "horror autotoxicus" to "gnothi seauton". Immunol. Today 12, 154-158.

Clackson, T. \& Wells, J. A. (1995). A hot spot of binding energy in a hormone-receptor interface, Science 267, 383-386.

Coutinho, A. (1986). Beyond clonal selection and network. Immunol. Rev. 110, 63-87.

De BoER, R. J. (1988). Symmetric idiotypic networks: connectance and switching, stability, and suppression. In: Theoretical Immunology, Part Two (Perelson, A. S. ed.) pp. 265-289. Reading, MA: Addison-Wesley.

De Boer, R. J. \& Hogeweg, P. (1989). Unreasonable implications of reasonable idiotypic network assumptions. Bull. Math. Biol. 51, 381-408.

De Boer, R. J. \& Perelson, A. S. (1991). Size and connectivity as emergent properties of a developing immune network. $J$. theor. Biol. 149, 381-424.

De Boer, R. J., Perelson, A. S. \& Kevrekidis, I. G. (1993a). Immune network behavior I: From stationary states to limit cycle oscillations. Bull. Math. Biol. 55, 745-780.

De Boer, R. J., Perelson, A. S. \& Kevrekidis, I. G. (1993b). Immune network behavior II: From oscillations to chaos and stationary states. Bull. Math. Biol. 55, 781-816.

De Boer, R. J. \& Perelson, A. S. (1993c). How diverse should the immune system be? Proc. R. Soc. Lond. B 252, 171-175.

De Boer, R. J., Boerlijst, M. C., Sulzer, B. \& Perelson, A. S. (1996). A new bell-shaped function for idiotypic interactions based upon crosslinking. Bull. Math. Biol. 58, 285-312.

Goodnow, C. C., Crosbie, J., Adelstein, S., Lavoie, T. B., Smith-Gill, S. J., BrinK, R. A., ET AL. (1988). Altered immunoglobulin expression and functional silencing of self-reactive B lymphocytes in transgenic mice. Nature 334, 676-682.

Grandien, A., Fucs, R., Nobrega, A., Andersson, J. \& Coutinho. A. (1995). Negative selection of multireactive B cell clones in normal adult mice. Eur. J. Immunol. 24, 1345-1352.

Herzenberg, L. A., Stall, A. M., Lalor, P. A., Sidman, C., Moore, W., Parks, D. R. \& Herzenberg, L. A. (1986). The Ly-1 B cell lineage. Immunol. Rev. 93, 81-102.

Holmberg, D., Forsgren, S., Ivars, F. \& Coutinho, A. (1984). Reactions among IgM antibodies derived from normal neonatal mice. Eur. J. Immunol. 14, 435-441.
Hoomjaas, H., Benner, R., Pleasants, J. R. \& Wostmann, B. S (1984). Isotypes and specificities of immunoglobulins produced by germ-free mice fed a chemically defined "antigen-free" diet. Eur. J. Immunol. 14, 1127-1130.

JANEWAY, C. A. \& TraVers, P. (1994). Immunobiology: the Immune System in Health and Disease. London: Current Biology Ltd.

JERNE, N. K. (1974). Towards a network theory of the immune system. Ann Immunol (Inst Pasteur) 125C, 373-389.

Kearney, J. F. \& VaKIL, M. (1986). Idiotype-directed interactions during ontogeny play a major role in the establishment of the adult B cell repertoire. Immunol. Rev. 94, 39-50.

Kearney, J. F., VAKil, M. \& Nicholson, N. (1987). Non-random $V_{H}$ expression and idiotype anti-idiotype expression in early B cells. In: Evolution and Vertebrate Immunity. The Antigen Receptor and MHC Gene Families (Kelsoe, G. \& Schulze, D. eds) pp. 175-190. Austin: University of Texas Press.

Lundkvist, I., Coutinho, A., Varela, F. \& Holmberg, D. (1989). Evidence for a functional idiotypic network among natural antibodies in normal mice. Proc. Natl. Acad. Sci. U.S.A. 86, 5074-5078

Malanchere, E., Marcos, M. A. R., Nobrega, A. \& Coutinho, A. (1995). Studies on the T cell dependence of natural IgM and IgG antibody repertoires in adult mice. Eur. J. Immunol. 25, $1358-1365$.

Nemazee, D. \& Burki, K. (1989). Clonal deletion of B lymphocytes in a transgenic mouse bearing anti-MHC class I antibody genes. Nature 337, 562-566.

Neumann, A. U. \& Weisbuch, G. (1992a). Window automata analysis of population dynamics in the immune system. Bull. Math. Biol. 54, 21-44.

Neumann, A. U. \& Weisbuch, G. (1992b). Dynamics and topology of idiotypic networks. Bull. Math. Biol. 54, 699-726.

Noest, A. J., TAKumi, K. \& De Boer, R. J. (1996). Pattern formation in B cell immune networks. (preprint).

Perelson, A. S. \& Oster, G. F. (1979). Theoretical studies of clonal selection: Minimal antibody repertoire size and reliability of self-nonself discrimination. J. theor. Biol. 81, 645-670.

Segel, L. A. \& Perelson, A. S. (1988). Computations in shape space: a new approach to immune network theory. In: Theoretical Immunology, Part Two (Perelson, A. S. ed.) pp. 321-343. Redwood City, CA: Addison-Wesley.

Stewart, J. \& Varela, F. (1991). Morphogenesis in shape space. Elementary meta-dynamics in a model of the immune network. J. theor. Biol. 153, 477-498.

Sulzer, B., Leo van Hemmen, J. \& Behn, U. (1994). Central immune system, the self and autoimmunity. Bull. Math. Biol. 56, 1009-1040.

Sulzer, B., De Boer, R. \& Perelson, A. S. (1996). Crosslinking reconsidered: Binding and crosslinking fields and the cellular response Biophysical Journal. 70, 1154-1168.

Weisbuch, G., De Boer, R. J. \& Perelson, A. S. (1990). Localized memories in idiotypic networks. J. theor. Biol. 146, 483-499.

Weisbuch, G., Santos, R. M. Z. D., Neumann, A. U. (1993). Tolerance to hormones and receptors in an idiotypic network model. J. theor. Biol. 163, 237-253.

\section{APPENDIX}

\section{Virgin, Immune, Suppressed}

For the case $K=1$ [Fig. 1(a, b, d)], we argued that clones can be referred to as immune or suppressed simply by checking their binding field. However, when $K<1$, or when a clone interacts with several ligands, this argument breaks down because the binding and the crosslinking field of each clone will differ. Here we generalize the argument about when a clone should be called immune or suppressed. 
Our theory is based upon the same concepts: a clone is suppressed when further stimulation decreases the rate of proliferation; otherwise it is immune. The proliferation rate, being determined by eqn (1), increases when the crosslinking field increases, and decreases when the binding field increases, i.e.

$$
\frac{\partial f\left(h, h_{x}\right)}{\partial h}<0, \quad \text { and } \quad \frac{\partial f\left(h, h_{x}\right)}{\partial h_{x}}>0 .
$$

Increasing the stimulation of a clone still further, by increasing the concentration of one of its ligands, will increase both $h$ and $h_{x}$ (with $\Delta h \geqslant \Delta h_{x}$ ).

Now consider a clone receiving $h=\sum_{i=1}^{n} K_{i} l_{i}$ and $h_{x}=\sum_{i=1}^{n} K_{i}^{2} l_{i}$ from $n$ different ligands $l_{i}$ with affinities $K_{i}$. To see when $f()$ decreases when $l_{i}$ increases, we solve $\partial f() / \partial l_{i}<0$ to obtain for each ligand

$$
h_{x}>K_{i}(1+h) / 2 \text {. }
$$

We now define a clone to be suppressed when eqn (A.2) is satisfied for its highest affinity, i.e. most stimulatory ligand. This definition implies that eqn (A.2) is satisfied for all ligands of a suppressed clone. We thus formulate our general suppression condition as

$$
h_{x}>\max _{i} K_{i}(1+h) / 2 .
$$

Assuming that any clone has a high affinity interaction, we can simplify eqn (A.3) to the special case that we used in Fig. 6.

$$
h_{x}>(1+h) / 2 \text {. }
$$

An alternative interpretation of the simplified suppression condition is to call a clone suppressed whenever it fails to respond to a new high affinity ligand, e.g. an antigen. Thus one could call eqn (A.3) a within network suppression condition and eqn (A.4) an external suppression condition. 